${ }^{1}$ Department of Pediatrics and Clinical Epidemiology, Sitaram Bhartia Institute of Science and Research, B-16, Qutab Institutional Area, New Delhi 110016, India

${ }^{2}$ Medical Research Council Epidemiology Resource Centre, University of Southampton, Southampton General Hospital, Southampton S016 6YD

Correspondence to:HPSSachdev hpssachdev@gmail.com

doi: 10.1136/bmj.39150.510475.AE

\title{
Effect of administration of intestinal anthelmintic drugs on haemoglobin: systematic review of randomised controlled trials
}

\author{
Anjana Gulani, attending consultant, Jitender Nagpal, attending consultant, ${ }^{1}$ Clive Osmond, senior \\ statistician, ${ }^{2}$ H P S Sachdev, senior consultant ${ }^{1}$
}

\section{ABSTRACT}

Objective To evaluate the effect of routine administration of intestinal anthelmintic drugs on haemoglobin. Design Systematic review of randomised controlled trials. Data sources Electronic databases and hand search of reviews, bibliographies of books, and abstracts and proceedings of international conferences.

Study selection Included studies were randomised or quasi-randomised controlled trials using an intestinal anthelmintic agent in the intervention group, in which haemoglobin was evaluated as an outcome measure. Trials in which treatment for schistosoma (praziquantel) was given exclusively to the intervention group were excluded.

Results The search identified 14 eligible randomised controlled trials. Data were available for 7829 subjects, of whom 4107 received an anthelmintic drug and 3722 received placebo. The pooled weighted mean difference (random effect model) of the change in haemoglobin was 1.71 (95\% confidence interval 0.70 to 2.73$) \mathrm{g} / \mathrm{l}$ ( $\mathrm{P}<0.001$; test for heterogeneity: Cochran $\mathrm{Q}=51.17, \mathrm{P}<0.001$; $I^{2}=61 \%(37 \%$ to $\left.76 \%)\right)$. With the World Health Organization's recommended haemoglobin cut-offs of $120 \mathrm{~g} / \mathrm{l}$ in adults and $110 \mathrm{~g} / \mathrm{l}$ in children, the average estimated reduction in prevalence of anaemia ranged from $1.1 \%$ to $12.4 \%$ in adults and from $4.4 \%$ to $21.0 \%$ in children. The estimated reductions in the prevalence of anaemia increased with lower haemoglobin cut-offs used to define anaemia.

Conclusions Routine administration of intestinal anthelmintic agents results in a marginal increase in haemoglobin $(1.71 \mathrm{~g} / \mathrm{l})$, which could translate on a public health scale into a small ( $5 \%$ to $10 \%$ ) reduction in the prevalence of anaemia in populations with a relatively high prevalence of intestinal helminthiasis.

\section{INTRODUCTION}

Anaemia is a widespread public health problem with major consequences for human health as well as social and economic development. The adverse health consequences associated with this malady include increased mortality in mothers and children with severe anaemia, impaired cognitive and physical development of children, and reduced work productivity of adults. ${ }^{1}$ Anaemia is estimated to affect nearly a third of the global population. ${ }^{2}$ It is more widespread in South Asia (53\%) than in other regions of the world. ${ }^{3}$ From a public health perspective, iron deficiency is believed to be the most important causal factor for anaemia. The fact that most anaemia control programmes, particularly in the developing world, rely on iron supplementation as the core strategy is therefore not surprising. Whether iron intervention alone can control anaemia on a public health scale is now, however, increasingly being questioned. A recent systematic review of randomised controlled trials of iron supplementation in children estimated that an average of between $38 \%$ and $62 \%$ of baseline anaemia (haemoglobin $<110 \mathrm{~g} / \mathrm{l}$ ) is responsive to iron supplementation among children aged under 6 ; the corresponding range for malarial hyperendemic regions is $6 \%$ to $32 \%{ }^{4}$

Administration of intestinal anthelmintic agents has been proposed as an additional intervention to reduce anaemia. Around two billion people globally are estimated to be infested with helminths, and 300 million of them have severe and permanent impairments. ${ }^{5}$ Observational data suggest an inverse relation between intestinal helminthiasis and haemoglobin concentrations. ${ }^{6}$ However, intervention trials using anthelmintic drugs have provided conflicting evidence; some authors have documented improvements in haemoglobin concentration, ${ }^{\mathrm{w} 1 \mathrm{w} 2}$ whereas other investigators have found no such benefit. ${ }^{\text {w3-w5 }}$ To aid public health decisions, we did a systematic review of randomised controlled trials to evaluate the effect of routine administration of intestinal anthelmintic agents on haemoglobin and identify any predictors of effect.

\section{METHODS}

\section{Searches}

We did Medline and extended Medline searches (1966 to 31 July 2006) by using the search words (haemoglobin OR hemoglobin OR anaemia OR anemia) AND (deworming OR anti-helminthic OR anthelminthic OR anthelminth OR mebendazole OR praziquantel OR pyrantel OR piperazine OR nitazoxanide OR levamisole OR albendazole OR bephenium OR niclosamide) with limits pertaining to "human" subjects for clinical trial, review, meta-analysis, and randomised 


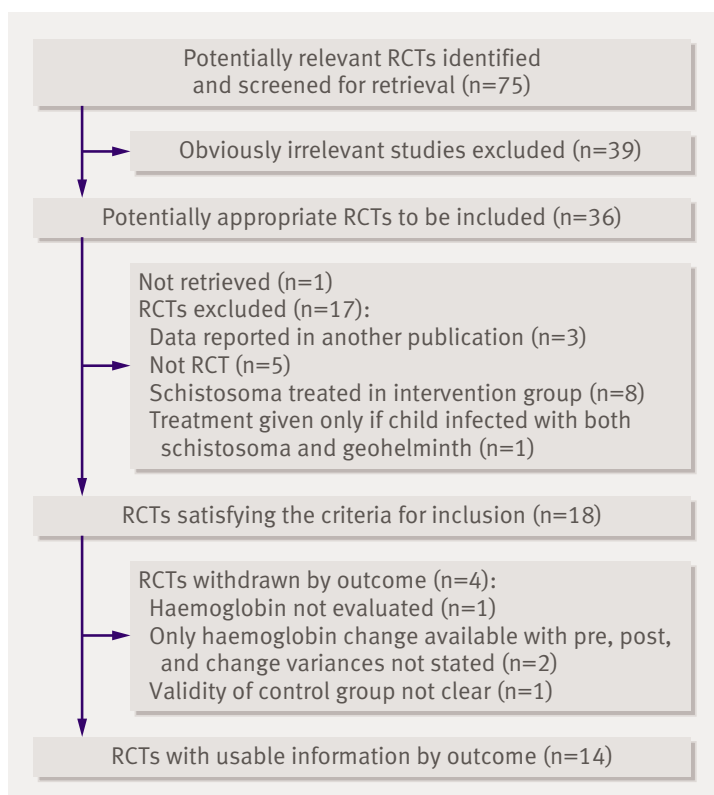

Fig 1 Trial flow for selection of randomised control trials to be included in meta-analysis. RCT=randomised controlled trial

controlled trial. We also did a similar search of the Cochrane controlled trials register. We imposed no language restrictions. We also reviewed reference lists of identified articles and hand searched reviews, bibliographies of books, and abstracts and proceedings of international conferences or meetings. We scanned the title and abstract of the trials identified in the computerised search to exclude studies that were obviously irrelevant. We scrutinised the full texts of the remaining studies and identified trials that fulfilled the inclusion criteria.

\section{Selection criteria}

To be included, trials had to be randomised or quasirandomised and controlled, use an intestinal anthelmintic drug in the intervention group, and evaluate haemoglobin as an outcome measure. We considered studies in which other micronutrients and drugs were simultaneously administered to be eligible if the only difference between the intervention and control groups was the intestinal anthelmintic drug. We excluded trials in which treatment for schistosoma (praziquantel given with or without laboratory confirmation) was given exclusively to the intervention group, in addition to the intestinal anthelmintic drug.

\section{Validity assessment}

We assessed the quality of trials by using recommended criteria. ${ }^{78}$ We classed concealment of allocation as adequate, unclear, or inadequate. To assess attrition, we classified studies by percentage of participants lost to follow-up (<4.9\%, 5-9.9\%, 10-19.9\%, and $\geq 20 \%$ ). For the purpose of this calculation, we considered the number of patients available at the last followup (at which data were retrievable). We classified blinding as double blinding, single blinding, no blinding, or unclear.

\section{Data abstraction}

$\mathrm{AG}$ and JN used pre-formed questionnaires to abstract the data in duplicate. The data included in this review were derived from the published papers or provided by the authors. If needed, and wherever possible, we contacted the authors for clarifications.

\section{Quantitative data synthesis}

For calculating pooled estimates, we needed the sample size, the mean change in haemoglobin or serum ferritin from the beginning to the end of the intervention, and the standard deviation of this change in the intervention and control groups. We used the following principles for derivations if actual variables were not stated: in a group, we assumed the lower of the two stated sample sizes at the beginning or end of a trial to be the sample size for the change; wherever feasible, we back calculated the standard deviation from the stated standard errors, $t$, or P values; wherever it was not stated, we calculated the mean change in the outcome variable as the difference between mean postintervention and pre-intervention values; and wherever it was not stated, we assumed the mean age of patients to be approximately equal to the median age, or the same as that of the entire study group.

The standard deviation for the change in haemoglobin was available or could be back calculated in several but not all trials. For the rest, we calculated this standard deviation by assuming correlations of 0.5 and 0 (independent) between the pre-intervention and postintervention variances. Considering the number of assumptions and calculations involved, to be confident about the interpretation we calculated four types of pooled estimates. In the first, we used the available values for the change. In the second and third, we calculated the standard deviation for the change for values that were missing or could not be back calculated, with the assumptions of a correlation $(\mathrm{p})=0.5$ or of independence $(p)=0$. For the fourth, we used the post-intervention scores and their respective standard deviations.

We evaluated the presence of publication bias in the extracted data by using funnel plots. ${ }^{9}$ We used the "metabias" command in Stata software to test funnel plots for asymmetry. We calculated the pooled estimates of the weighted mean difference of the evaluated change in outcome variable between the control and intervention group by both fixed effects and random effects model assumptions by using the "metan" command in Stata software. We mainly report random effects estimates here, because most of the pooled results obtained were statistically heterogeneous.

We carried out prespecified stratified analyses for age group, developing or developed country, malaria endemicity, schistosoma endemicity, pre-intervention worm load, methodological quality, compliance monitoring, number of anthelmintic courses, co-administration of iron, baseline haemoglobin concentrations, 


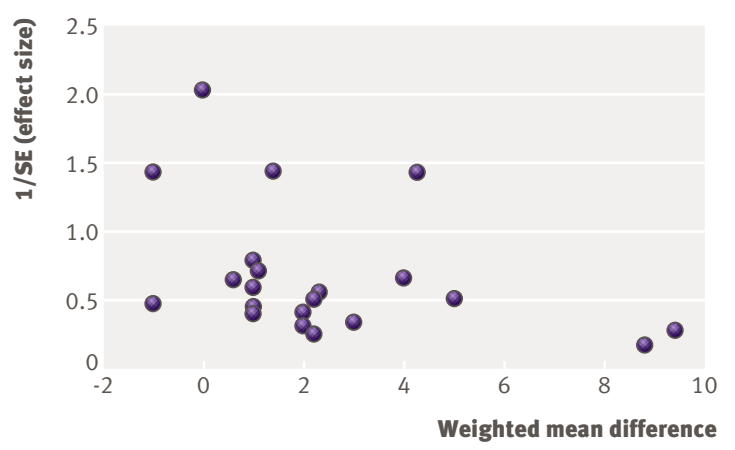

Fig 2 Funnel plot of haemoglobin, with unknown standard deviations derived with assumption $(p)=0.5$

and baseline anthropometry (in children). We also explored the contribution of these variables to heterogeneity by meta-regression with the "metareg" command in Stata software with the restricted maximum likelihood option. In the study in which one control group was used for two intervention groups, the estimates of treatment effect were thus correlated. We explicitly modelled this correlation in obtaining the maximum likelihood estimate of the treatment effect pooled across all studies.

\section{RESULTS}

We identified 36 potentially eligible randomised controlled trials. Twenty two studies were ineligible ${ }^{\mathrm{w} 6-\mathrm{w} 27}$ (fig 1). We therefore evaluated 14 trials in this systematic review. ${ }^{\text {w1-w5 w28-w36 }}$

\section{Study characteristics}

Table A on bmj.com summarises the baseline characteristics of the included trials. These studies were primarily done in developing countries (five in South Asia and nine in Africa) on pre-schoolchildren and schoolchildren (11/14). One study was done in non-pregnant adult women, one in pregnant women, and one in all age groups. Ten of the studies used albendazole as the anthelmintic drug, three used mebendazole, and one used bephenium. Iron was used as co-intervention in more than half of the studies (7/12). Twelve studies were done in areas classified as endemic for malaria, and six were done in areas endemic for schistosoma.

\section{Quantitative data synthesis}

We found no evidence of asymmetry of the funnel plot (fig 2), suggesting an absence of publication bias. We confirmed this by using the Egger's (weighted regression) method ( $\mathrm{P}$ for bias=0.11) and the Begg's (rank correlation) method (continuity corrected $\mathrm{P}=0.11$ ).

Data were available for 7829 patients, of whom 4107 received deworming treatment and 3722 received placebo. The pooled weighted mean difference (random effects model) of the change in haemoglobin (pre-intervention to post-intervention difference) after deworming was 1.71 (95\% confidence interval 0.70 to 2.73$) \mathrm{g} / \mathrm{l}$ $(\mathrm{P}<0.001$; test for heterogeneity: Cochran $\mathrm{Q}=51.17$, $\mathrm{P}<0.001 ; I^{2}=61 \%$, $(37 \%$ to $76 \%)$ ) (fig 3 , table $\mathrm{B}$ on bmj.com). The results were similar when we calculated the missing standard deviations by assuming independence and with post-intervention scores (independence: weighted mean difference 1.77 (0.75 to 2.80) $\mathrm{g} / \mathrm{l}, \quad \mathrm{P}=0.001$; post-intervention scores: weighted mean difference 2.01 ( 0.58 to 3.44$) \mathrm{g} / \mathrm{l}, \mathrm{P}=0.006)$. The effect size was marginally higher when we restricted the analysis to those studies with available standard deviation scores for the change in haemoglobin (weighted mean difference 2.55 (1.52 to 3.57 ) g/l, $\mathrm{P}<0.001$; test for heterogeneity: Cochran $\mathrm{Q}=19.89$, $\mathrm{P}=0.225 ; I^{2}=20 \%(0 \%$ to $\left.55 \%)\right)$.

Other markers of iron status were estimated in only three of the studies, ${ }^{\text {w5 }}$ w30 w31 which precluded a formal meta-analysis. Also, one study did not make it clear whether serum ferritin concentration was depicted as the arithmetic or geometric mean. ${ }^{\text {w30 }}$ Deworming increased the serum ferritin and erythrocyte

\begin{tabular}{|c|c|c|c|c|}
\hline \multirow[b]{2}{*}{ Study characteristic } & \multicolumn{2}{|c|}{ Univariate analysis } & \multicolumn{2}{|c|}{ Controlling for all variables $\dagger$} \\
\hline & WMD $(95 \% \mathrm{Cl})$ & $\mathbf{P}$ & WMD $(95 \% \mathrm{Cl})$ & $\mathbf{P}$ \\
\hline \multicolumn{5}{|l|}{ Study quality: } \\
\hline Allocation concealment (not adequate $v$ adequate) & $0.98(-0.96$ to 2.92$)$ & 0.321 & $-0.80(-5.92$ to 4.32$)$ & 0.759 \\
\hline Attrition $(>10 \% v<10 \%)$ & $0.81(-1.14$ to 2.76$)$ & 0.414 & $0.41(-1.96$ to 2.78$)$ & 0.735 \\
\hline Blinding (not double blind $v$ double blind) & $-0.72(-2.92$ to 1.50$)$ & 0.527 & $-1.54(-5.11$ to 2.04$)$ & 0.400 \\
\hline Adults included $v$ children only & $2.85(1.00$ to 4.70$)$ & 0.002 & $3.24(-0.40$ to 6.87$)$ & 0.081 \\
\hline Malaria hyperendemic $v$ not & $0.35(-2.00$ to 2.70$)$ & 0.770 & $3.04(-2.80$ to 8.88$)$ & 0.307 \\
\hline Schistosoma hyperendemic $v$ not $(n=19)$ & $-0.72(-2.62$ to 1.18$)$ & 0.460 & $\mathrm{NI}$ & \\
\hline Worm load high $v$ low $(n=20)$ & $0.76(-1.06$ to 2.58$)$ & 0.414 & $-0.62(-4.95$ to 3.72$)$ & 0.781 \\
\hline Unit increase in No of anthelmintic courses $(n=20)$ & $-0.49(-1.13$ to 0.15$)$ & 0.132 & $\mathrm{NI}$ & \\
\hline Iron co-intervention $v$ none & $1.92(0.22$ to 3.62$)$ & 0.027 & $1.16(-1.59$ to 3.90$)$ & 0.408 \\
\hline Unit increase in mean baseline haemoglobin status $(\mathrm{g} / \mathrm{l})$ & $0.04(-0.06$ to 0.14$)$ & 0.387 & $0.14(-0.08$ to 0.36$)$ & 0.221 \\
\hline Unit increase in weight for age $z$ score $(n=15)$ & $1.45(-0.40$ to 3.29$)$ & 0.124 & $\mathrm{NI}$ & \\
\hline
\end{tabular}

$\mathrm{Nl}=$ not included in multivariate analyses as number of variables that can be included is limited and information missing in some variables.

*Calculations done by standard deviation calculated with assumption $(p)=0.5$.

†Sample size for multivariate analysis is 20 analytic units. 


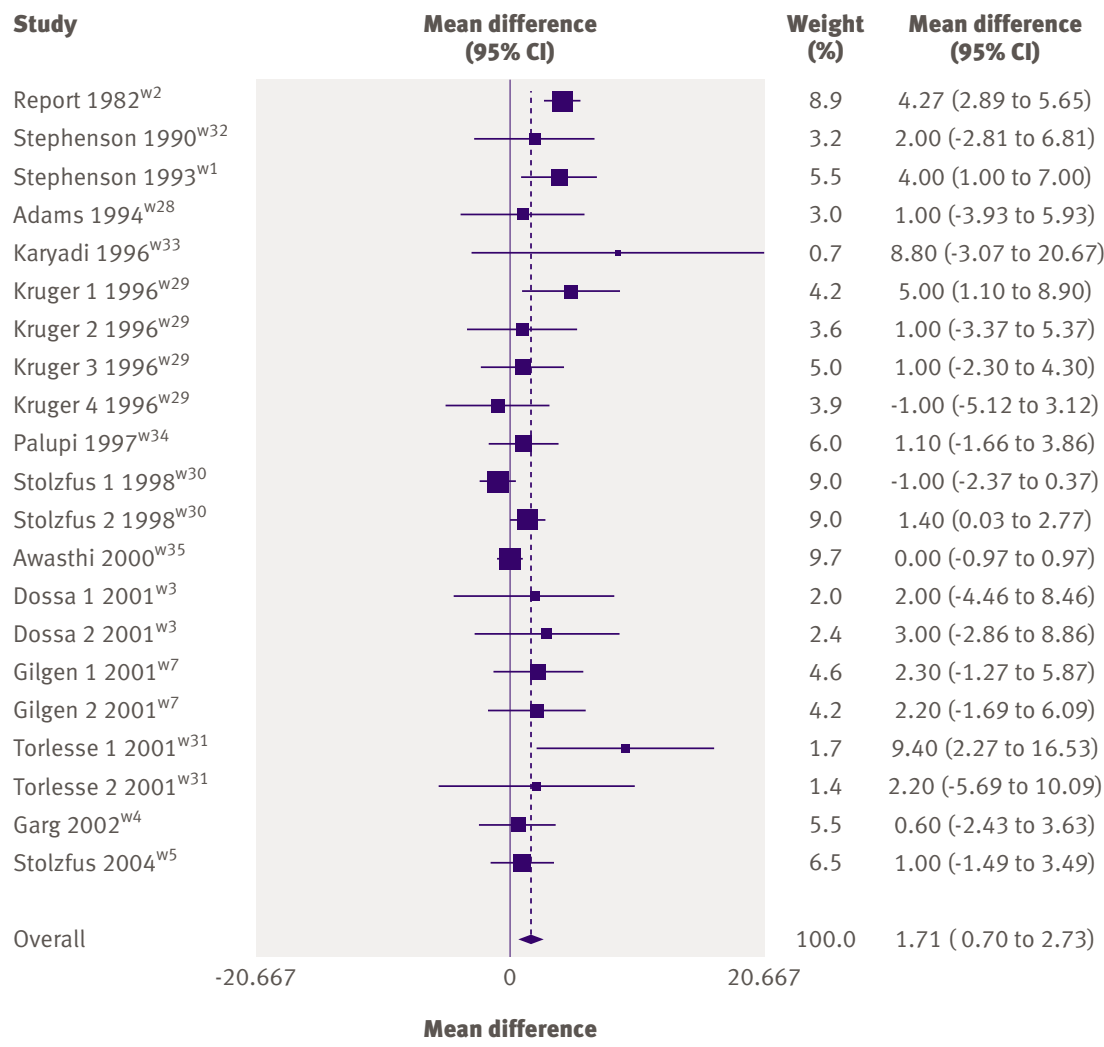

Fig 3 | Forest plot of haemoglobin with unknown standard deviations derived with assumption $(p)=0.5$

protoporphyrin concentration in pre-schoolchildren and schoolchildren in Zanzibar after 12 months of anthelmintic treatment. ${ }^{\text {w5 }}$ w0 The third study included pregnant women and documented a decrease in mean serum ferritin concentration from the baseline to the third trimester in all patients. ${ }^{\text {w31 }}$ However, use of albendazole did not result in any significant increase in serum ferritin concentrations.

Sensitivity analyses (table B on bmj.com) suggested a greater rise in haemoglobin (non-overlapping confidence intervals) in trials that included adults. Metaregression (table 1) by univariate analysis suggested that inclusion of adults and use of iron as a co-intervention were significant predictors of a positive effect of the deworming agent. However, on multivariate

Table 2 | Estimated effects of deworming in reducing baseline anaemia prevalence (\%) as defined by various cut-offs

\begin{tabular}{|c|c|c|c|c|}
\hline \multirow[b]{2}{*}{ Assumption } & \multicolumn{4}{|c|}{ Haemoglobin ( $\mathrm{g} / \mathrm{l}$ ) cut-off for defining anaemia } \\
\hline & 90 & 100 & 110 & 120 \\
\hline \multicolumn{5}{|c|}{ Low haemoglobin response } \\
\hline Range & $4.5-17.7$ & $3.0-13.3$ & $1.8-9.0$ & $0.4-5.1$ \\
\hline Mean (SE) & $9.4(0.6)$ & $6.5(0.5)$ & $4.0(0.3)$ & $2.1(0.2)$ \\
\hline \multicolumn{5}{|c|}{ Average haemoglobin response } \\
\hline Range & $10.9-38.3$ & $7.4-29.9$ & $4.4-21.0$ & 1.1-12.4 \\
\hline Mean (SE) & $21.5(1.3)$ & $15.4(1.1)$ & $9.7(0.8)$ & $5.1(0.5)$ \\
\hline \multicolumn{5}{|c|}{ High haemoglobin response } \\
\hline Range & 17.1-54.2 & $11.8-43.8$ & $7.2-32.0$ & $2.0-19.6$ \\
\hline Mean (SE) & $32.2(1.8)$ & $23.6(1.5)$ & $15.3(1.2)$ & $8.3(0.8)$ \\
\hline
\end{tabular}

analysis, neither of these variables was identified as a significant predictor.

We also estimated the average expected reduction in the prevalence of anaemia with deworming on the basis of the calculated weighted mean difference by using varying haemoglobin cut-offs to define anaemia (table 2). With the World Health Organization's recommended haemoglobin cut-offs of $120 \mathrm{~g} / 1$ in adults and $110 \mathrm{~g} / \mathrm{l}$ in children, the average estimated reduction in the prevalence of anaemia ranged from $1.1 \%$ to $12.4 \%$ in adults and from $4.4 \%$ to $21.0 \%$ in children. The estimated reductions in the prevalence of anaemia increased with lower haemoglobin cutoffs used to define anaemia.

\section{DISCUSSION}

The results from these largely heterogeneous data derived from randomised controlled trials show that deworming without previous screening marginally improves haemoglobin concentration (weighted mean difference 1.71 (95\% confidence interval 0.70 to 2.73$) \mathrm{g} / \mathrm{l}, \mathrm{P}<0.001)$. Inclusion of adults and coadministration of iron emerged as significant predictors of greater haemoglobin response and heterogeneity requiring further exploration. The projections of expected average reductions in baseline anaemia through routine deworming ranged from 5\% to $10 \%$. The estimated reduction in the prevalence of anaemia was higher with lower haemoglobin cut-offs.

\section{Strengths and limitations}

The main conclusion about the rise in haemoglobin after routine administration of intestinal anthelmintic agents remained stable over a large spectrum of sensitivity analyses. Influence analysis - namely, the effect of omitting one study at a time (data not shown) — did not reveal an overwhelming effect of any single trial.

Several limitations merit consideration. Firstly, most of the trials did not specifically evaluate the iron status of the patients. Secondly, in trials with missing data on the variability of the change in haemoglobin, we made several imputations on the basis of the prespecified assumptions. The sensitivity analysis suggested that these imputations were robust. Finally, we did multiple subgroup and meta-regression analyses for important prespecified variables, which increased the possibility of false positive results. The identified significant predictors of greater haemoglobin response and heterogeneity should therefore be considered as only exploratory in nature, rather than definitive.

\section{Implications}

A few interesting observations emerged that have programmatic implications and can provide direction for future research. Information on iron status was provided in only three studies. In the two studies done in children, deworming increased the serum ferritin and protoporphyrin concentrations, ${ }^{\mathrm{w} 5 \mathrm{w} 30}$ whereas the study in pregnant women found no change in the iron status. ${ }^{\text {w31 }}$ The physiological changes induced by pregnancy, including excessive demand for iron, may have 


\section{WHAT IS ALREADY KNOWN ON THIS TOPIC}

Anaemia is a widespread public health problem with major consequences for human health, as well as for social and economic development

Iron deficiency is believed to be the most important causal factor for anaemia, but whether iron intervention alone can control anaemia on a public health scale is questionable

Administration of intestinal anthelmintic drugs has been proposed as an additional intervention to reduce anaemia

\section{WHAT THIS STUDY ADDS}

Routine administration of intestinal anthelmintic drugs results in a marginal increase in haemoglobin concentration

On a public health scale, this could translate into a small (5\% to $10 \%)$ reduction in the prevalence of anaemia in populations with a relatively high prevalence of intestinal helminthiasis routine administration of anthelmintics. Albendazole and mebendazole, the drugs used in most of the studies, are generally regarded as safe when used in anthelmintic doses. ${ }^{12}$ Side effects such as gastrointestinal upset, headache, dizziness, alopecia, hypersensitivity, leucopenia, pancytopenia, and elevation of liver enzymes with albendazole and gastrointestinal upset and hypersensitivity with mebendazole have been rarely reported in the literature. ${ }^{1314}$ The reporting of adverse effects in the included trials was unfortunately too poor to provide relevant evidence. Economic considerations of routine anthelmintic treatment, particularly in combination with iron prophylaxis, are also important. In the absence of specific data, commenting on this aspect would be difficult; however, with the projected reductions in the prevalence of anaemia, the cost effectiveness would be unlikely to be substantial.

prevented a relative increase in indicators of iron in the dewormed group in this study. The studies in children suggest that routine deworming may be increasing haemoglobin by enhancing iron status, possibly by preventing helminth induced blood loss. ${ }^{10}$ However, further data are needed in this context, and future studies could usefully incorporate indicators of iron status in serum and of iron and blood losses in stool.

Surprisingly, the baseline helminthic prevalence did not emerge as a significant predictor of haemoglobin response. This may have been because the egg density would be a better quantification of the helminthic load than single prevalence data. However, these data were not available in all the studies to allow a pertinent analysis. Alternatively, the host could have dynamically regulated the iron absorption in relation to the presence or absence of intestinal helminths.

An increase in the number of doses of anthelmintic agent was not a significant predictor of haemoglobin response. Further trials could include information on the time sequence of helminthic reinfection and haemoglobin concentrations to gain better insight into this observation. Furthermore, we need to remember that this observation does not stem from a head to head comparison of single versus multiple doses of anthelmintic drug. Another systematic review also could not document a greater effect on mean weight change in children with multiple doses of anthelmintics. ${ }^{11}$

The programmatic implications of these findings should be examined. Projections suggested that this marginal increase in haemoglobin could translate into a small $(5 \%$ to $10 \%)$ reduction in the prevalence of anaemia on a public health scale. Better returns may occur in adults and in populations receiving iron supplementation and with a high prevalence of intestinal helminthiasis. However, in this context, no leads emerge regarding the optimal frequency and periodicity of anthelmintics. Additional important aspects influencing public health decisions would include evaluation of other benefits such as growth and cognitive performance, ${ }^{11}$ ethical dimensions of prescribing anthelmintic agents in populations with low or varying prevalence of infestations, and adverse effects of

\section{Conclusion}

Routine administration of intestinal anthelmintic drugs results in a marginal increase in haemoglobin $(1.71 \mathrm{~g} / \mathrm{l})$, which could translate on a public health scale into a small (5\% to $10 \%)$ reduction in the prevalence of anaemia in populations with a relatively high prevalence of intestinal helminthiasis.

We acknowledge the efforts of Nidhi Gupta in helping with the search and retrieval of the full text of the articles.

Contributors: AG and JN prepared the protocol, applied the search strategy, retrieved the articles, and extracted data. CO contributed to the statistical analysis. HPSS developed the idea for review, finalised the protocol and search strategy, and did the statistical analysis. All authors contributed to the drafting of the final version of the paper. HPSS is the guarantor.

Funding: Sitaram Bhartia Institute of Science and Research, New Delhi, India. The funding source had no involvement in the study or the decision to publish the manuscript.

Competing interests: None declared.

Ethical approval: Not needed.

1 Joint statement by the World Health Organization and the United Nations Children's Fund. Focusing on anaemia: towards an org/English/AD/FCH/NU/WHO04_Anemia.pdf.

2 United Nations Administrative Committee on Coordination SubCommittee on Nutrition (ACC/SCN). Fourth report on the world nutrition situation. Geneva: ACC/SCN, International Food Policy Research Institute, 2000.

3 Mason JB, Lotfi M, Dalmiya N, Sethuraman K, Deitchler M, Geibel S et al. The micronutrient report: current progress and trends in the control of vitamin $A$, iodine and iron deficiencies. Ottawa: Micronutrient Initiative, 2001. (Available at www.micronutrient.org.)

4 Gera T, Sachdev HPS, Nestel P, Sachdev SS. Effect of iron supplementation on hemoglobin response in children: systematic review of randomized control trials. J Pediatr Gastroenterol Nutr 2007;44:468-86.

5 World Health Organization. The millennium development goals deworming. Geneva: WHO, 2005. (WHO/CDS/CPE/PVC/2005.1.)

6 Ezeamama AE, Friedman JF, Olveda RM, Acosta LP, Kurtis JD, Mor V, et al. Functional significance of low-intensity polyparasite helminth infections in anemia. J Infect Dis 2005;192:2160-70.

7 Higgins JPT, Green S, eds. Assessment of study quality. Cochrane Handbook for Systematic Reviews of Interventions 4.2.6 [updated September 2006]; Section 6. In: The Cochrane Library, Issue 4. Chichester: John Wiley \& Sons, 2006.

8 Juni P, Altman DJ, Egger M. Assessing the quality of randomized controlled trials. In: Egger M, Smith GD, Altman DG, eds. Systematic reviews in health care: meta-analysis in context. London: $\mathrm{B}$ M) Publishing, 2001:87-108.

9 Sterne JAC, Egger M, Smith GD. Investigating and dealing with publication and other biases. In: Egger M, Smith GD, Altman DJ, eds. integrated approach for effective anaemia control. 2004. www.paho. 
Systematic reviews in health care: meta-analysis in context. London: BMJ Publishing, 2001:189-208.

10 Stephenson LS. Impact of helminth infections on human nutrition. New York: Oxford University Press, 1993:131-60.

11 Dickson R, Awasthi S, Williamson P, Demellweek C, Garner P. Effects of treatment for intestinal helminth infection on growth and cognitive performance in children: systematic review of randomised trials. $B M$ J 2000;320:1697-701.

12 World Health Organization. Report of the WHO informal consultation on the use of praziquantel during pregnancy/lactation and albendazole/mebendazole in children under 24 months. Geneva: WHO, 2002. (WHO/CDC/CPE/PVC/2002.4.)

13 Royal College of Paediatrics and Child Health (UK) and Neonatal and Paediatrics Pharmacists Group (UK). Medicines for children. 1st Indian edition. New Delhi: B I Publications, 2004:14.

14 Royal College of Paediatrics and Child Health (UK) and Neonatal and Paediatrics Pharmacists Group (UK). Medicines for children. 1st Indian edition. New Delhi: B I Publications, 2004:379.

Accepted: 28 February 2007 